\title{
CDMA Multiuser Detection: A Nonlinear Programming Approach
}

\author{
Aylin Yener, Member, IEEE, Roy D. Yates, Member, IEEE, and Sennur Ulukus, Member, IEEE
}

\begin{abstract}
The optimum receiver to detect the bits of multiple code-divison multiple access (CDMA) users has exponential complexity in the number of active users in the system. Consequently, many suboptimum receivers have been developed to achieve good performance with less complexity. In this paper, we take the approach of approximating the solution of the optimum multiuser detection problem (OMUD) using nonlinear programming relaxations. First, we observe that some popular suboptimum receivers indeed correspond to relaxations of the optimal detection problem. In particular, one proposed approximation method yields to iterative solutions which correspond to previously proposed heuristic nonlinear detectors. Using a nonlinear programming approach, we identify the convergence properties of these iterative detectors. Secondly, we propose a relaxation that yields a receiver which we call the generalized minimum mean squared error detector. We give a simple iterative implementation of the detector. Its performance is evaluated and comparisons to other suboptimum detection schemes are given.
\end{abstract}

Index Terms-Approximation algorithms, code division multiple access (CDMA), interference cancellation, multiuser detection, nonlinear programming.

\section{INTRODUCTION}

I T HAS been long known that the near-far problem of multiuser code-divison multiple access (CDMA) systems is not inherent to the code-division access method but to the fact that the matched filter receiver designed for a single-user Gaussian channel is not optimum for the multiple-user CDMA channel [1]. Further, optimum detection of multiple users' bits has been shown to be an NP hard problem [2]. This observation resulted in the development of many suboptimum receivers that have reasonable complexity with near-optimum performance [3]-[6]. These suboptimum receivers have been motivated by several criteria. Among the most popular linear detectors are the decorrelator [3] and the minimum mean squared error (MMSE) receiver [4]. The decorrelator suppresses the multiple access interference (MAI) totally while enhancing the Gaussian noise and is the optimum detector if the received powers of the users are unknown

Paper approved by G. E. Corazza, the Editor for Spread Spectrum of the IEEE Communications Society. Manuscript received January 12, 2000; revised August 26, 2001, and October 28, 2001. This work was presented in part at the 33rd Asilomar Conference on Signals, Systems and Computers, Pacific Grove, CA, October 1999

A. Yener is with the Electrical Engineering Department, The Pennsylvania State University, University Park, PA 16802-2705 USA (e-mail: yener@ ee.psu.edu).

R. D. Yates is with WINLAB, Rutgers University, Piscataway, NJ 088548060 USA (e-mail: ryates@winlab.rutgers.edu).

S. Ulukus is with the Department of Electrical and Computer Engineering, University of Maryland, College Park, MD 20742 USA (e-mail: ulukus@ eng.umd.edu).

Publisher Item Identifier S 0090-6778(02)05548-4. at the receiver [1]. The MMSE receiver [4] gives the minimum mean squared error between the filter output and the transmitted bit and also maximizes the output signal-to-interference ratio. Both detectors are optimum when no noise is present. Decision-directed nonlinear detectors that generally result in lower bit error rates (BERs) have been proposed in [5] and [6]. The decorrelating decision feedback detector [6] cancels the interference from the users that have already been decoded, and suppresses interference from users that have not yet been decoded, to obtain the decision statistic for the current user. The multistage detector [7] uses the previous stage bit estimates of all interferers to reconstruct and cancel the interference to the current user in the current stage. The interference canceler in [8] subtracts the interference estimate of the previously decoded users from the matched filter output for the current user and uses this decision statistic to decode the bit of the current user.

Our aim in this study is to approach the optimum multiuser detection problem from a nonlinear programming point of view. The original optimum multiuser detection problem (OMUD) is a $0-1$ quadratic program for which there exists no efficient algorithm. The general approach in the presence of such hardship is to approximate the solution by working on an easier problem that can be solved efficiently. The easier problem to be solved is a relaxation of the original problem. The relaxed solution is then mapped to the solution set of the original problem, ideally arriving at a near-optimum solution. For the multiuser detection problem, a relaxation corresponds to a near-optimum multiuser detector.

Using a nonlinear programming approach, we observe that some popular suboptimum detectors are relaxed solutions, i.e., approximations to the optimum detection problem. This approach helps us understand the previously unidentified convergence properties of some known iterative nonlinear detectors. Furthermore, a new relaxation method is proposed that results in a simple iterative detector whose performance is then evaluated.

\section{OMUD AND ITS RELAXATIONS}

We consider a synchronous CDMA system employing BPSK modulation. The received signal is given by

$$
r(t)=\sum_{i=1}^{N} \sqrt{q_{i}} a_{i} s_{i}(t)+n(t)
$$

where $N$ is the number of users, $q_{i}$ and $a_{i}$ are received power and the transmitted bit ( \pm 1 equiprobably), and $s_{i}(t)$ is the unit energy signature, i.e., $\int_{0}^{T} s_{i}(t) s_{i}(t) d t=1$, of user $i$, and $n(t)$ is 
the additive white Gaussian noise (AWGN) process with power spectral density $\sigma^{2}$. The matched filter output of the $i$ th user is given by $y_{i}=\int_{0}^{T} r(t) s_{i}(t) d t$. The received signal vector at the output of the matched filters, $\boldsymbol{y}$, is a sufficient statistic for the multiuser detection problem and is given by

$$
\boldsymbol{y}=\boldsymbol{\Gamma} \Lambda \boldsymbol{a}+\boldsymbol{n}
$$

where $\boldsymbol{\Gamma}$ is the nonnegative definite cross-correlation matrix with $\Gamma_{i j}=\int_{0}^{T} s_{i}(t) s_{j}(t) d t, \Lambda$ is a diagonal matrix containing the users' received amplitudes $\Lambda_{i i}=\sqrt{q_{i}}, \boldsymbol{a}$ is the vector containing the information bits of the users, and $\boldsymbol{n}$ is a zero mean Gaussian random vector with auto covariance matrix $E\left[n n^{\top}\right]=$ $\sigma^{2} \mathbf{\Gamma}$.

The aim of multiuser detection is to recover the information bits, $\boldsymbol{a}$. The solution of the OMUD problem [1], [3], [9] employs maximum-likelihood estimation. The optimum estimate for the bit vector is $\boldsymbol{a}^{*}$, if $\boldsymbol{a}^{*}$ minimizes the cost function which is quadratic in $\boldsymbol{a}$. Specifically,

$$
\boldsymbol{a}^{*}=\arg \min _{\boldsymbol{a} \in\{-1,1\}^{N}} \boldsymbol{a}^{\top} \boldsymbol{R} \boldsymbol{a}-2 \boldsymbol{a}^{\top} \boldsymbol{\Lambda} \boldsymbol{y}
$$

where $\boldsymbol{R}=\boldsymbol{\Lambda} \boldsymbol{\Gamma} \boldsymbol{\Lambda}$ with $R_{i j}=\sqrt{q_{i}} \sqrt{q_{j}} \Gamma_{i j}$. Although it has been shown recently that certain special $R$ structures allow construction of polynomial time algorithms to find the optimum solution [10], [11], the problem for general correlation matrices remains NP hard and one can find the optimum $\boldsymbol{a}$ only by an exhaustive search of $2^{N}$ candidate vectors [2].

It is interesting to note that this model can also be applied to detection on a multiple bit window in an asynchronous CDMA system with multipath fading [12]. If the arrival times of the $L$ propagation paths are known, it is sufficient to observe the outputs of the $N \times L$ matched filters for all paths of all users over the length of the window. Recently, it has also been shown that when antenna arrays are employed at the receiver, the sufficient statistics are obtained by passing the received signal through beamformers directed to each path of each user and finally combining them with a bank of $N$ RAKE combiners [13]. For each of these models, with careful definition of cross correlations between the signatures of all users over the multiple bit windows, one can construct the corresponding $\boldsymbol{R}$ matrix and the optimum detection problem can be shown to be of the form of (3); see, for example, [13, eq. (19)]. Thus, the methodology and the resulting algorithms we use in this work remain valid for the most general model.

In this work, we will concentrate on cases where the signatures of the users are linearly independent and $\boldsymbol{\Gamma}$ and hence $R$ are positive definite. In this case, the objective function in (3) is strictly convex in $\boldsymbol{a}$ and has a well-defined unique minimizer over a convex set [14]. Thus, we can find solutions by relaxing the constraint set-which in the original problem contains only the corners of the unit hypercube-such that the resulting "relaxed" constraint set is convex. Fig. 1 shows the different relaxed constraint sets for the two-user case. Three basic relaxations that we will consider in the sequel are: relaxation of the constraint set to the whole unit hypercube (region I), relaxation of the constraint set to the sphere which covers the unit hypercube (regions $\mathrm{I}+\mathrm{II}$ ), and relaxation to the whole space (regions $\mathrm{I}+\mathrm{II}+\mathrm{III})$. Note that we require the constraint set for each re-

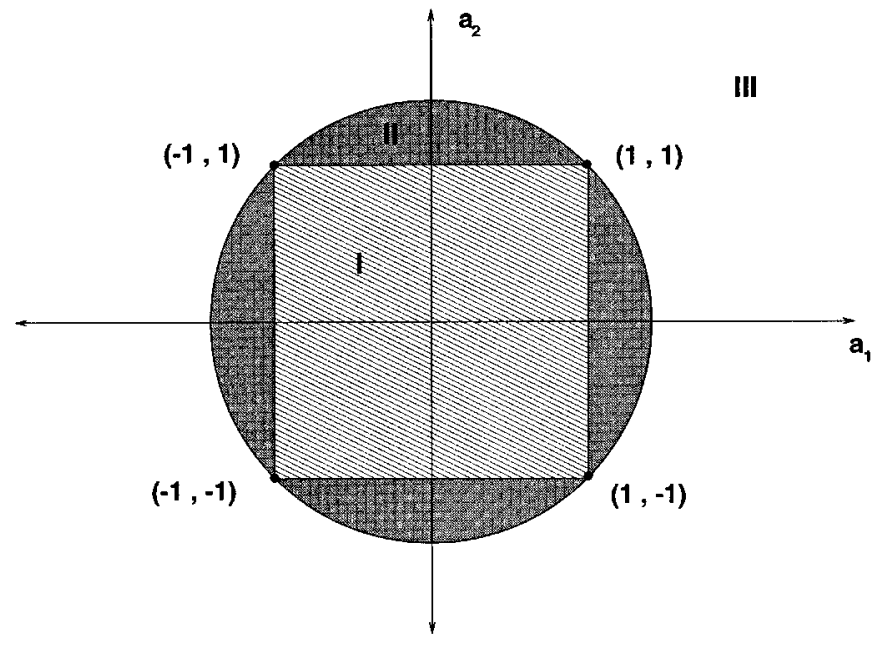

Fig. 1. Relaxed constraint sets for the two-user system which yield the following detectors: I-soft interference canceler (Section IV), I+II-generalized MMSE (Section V), I+II+III-decorrelator (Section III).

laxation to contain the feasible set of the original problem. The solution can then be mapped to the feasible set of the original problem by taking the sign of each component of the relaxed solution vector (since bits are equiprobably \pm 1 ).

\section{DECORRELATOR}

We first consider the simplest relaxation, where the feasible set is relaxed to contain the $N$ dimensional space $\mathcal{R}^{N}$

$$
\min _{\boldsymbol{a} \in \mathcal{R}^{N}} \boldsymbol{a}^{\top} \boldsymbol{R} \boldsymbol{a}-2 \boldsymbol{a}^{\top} \boldsymbol{\Lambda} \boldsymbol{y}
$$

This relaxation effectively removes the constraints and converts the discrete optimization problem into a continuous one. Since the cost function is convex in its variable, this problem has a unique minimum

$$
\hat{\boldsymbol{a}}=\boldsymbol{R}^{-1} \boldsymbol{\Lambda} \boldsymbol{y}=\boldsymbol{\Lambda}^{-1} \boldsymbol{\Gamma}^{-1} \boldsymbol{y}=\boldsymbol{a}+\boldsymbol{\Lambda}^{-1} \boldsymbol{\Gamma}^{-1} \boldsymbol{n} .
$$

Taking the sign of the solution vector yields the well-known decorrelating detector [3]. Note that, due to the sign operation, the detector is insensitive to signal amplitudes, i.e., the bit estimate resulting from $\hat{\boldsymbol{a}}$ is the same as that of $\boldsymbol{\Lambda} \hat{\boldsymbol{a}}=\boldsymbol{\Gamma}^{-1} \boldsymbol{y}$.

\section{SOFt INTERfERENCE CANCELLATION}

The constraint set of the optimum multiuser detection problem (3) consists of the corner points of the unit hypercube. An effective approximation method is to relax the constraint set to cover the whole hypercube and use nonlinear programming algorithms to find the solution of the new convex programming problem [15]. The relaxed problem is

$$
\boldsymbol{a}^{*}=\arg \min _{\boldsymbol{a} \in[-1,1]^{N}} \boldsymbol{a}^{\top} \boldsymbol{R} \boldsymbol{a}-2 \boldsymbol{a}^{\top} \boldsymbol{\Lambda} \boldsymbol{y}
$$

Both the cost function and the constraint set in (6) are convex. Thus, (6) has a unique minimum. However, the optimum point does not have a closed form and one should use iterative methods to find the solution. One class of iterative methods that can be used is the class of constrained gradient methods. Further, the simplicity of the constraint set, i.e., the fact that it has 


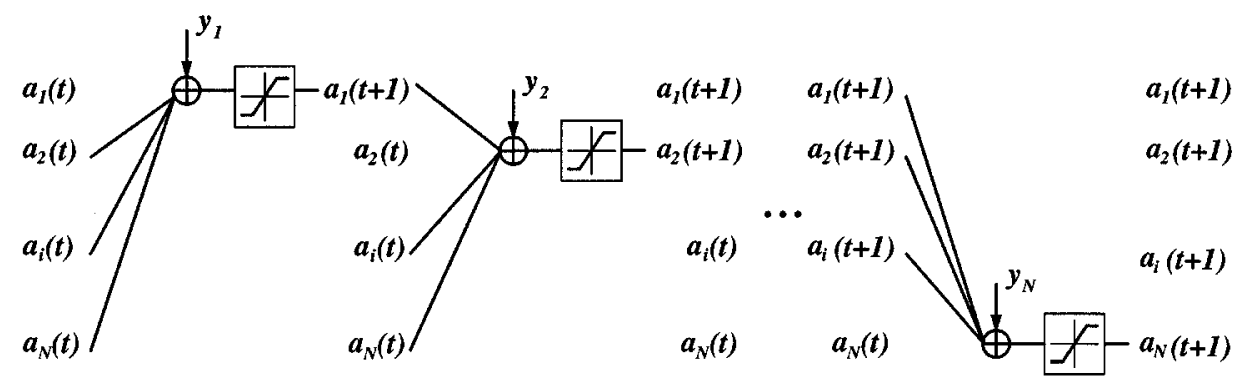

Fig. 2. Successive soft interference canceler; see (9) and (11).

a Cartesian product form, enables us to define special iterative projection algorithms [14]. In particular, the following two algorithms, the nonlinear Gauss-Seidel and the nonlinear Jacobi algorithms, respectively, converge to the minimum of (6) under certain conditions. Let $g\left(a_{1}, \ldots, a_{N}\right)=\boldsymbol{a}^{\top} \boldsymbol{R} \boldsymbol{a}-2 \boldsymbol{a}^{\top} \boldsymbol{\Lambda} \boldsymbol{y}$ denote the function to be minimized. In the Gauss-Seidel iteration, $a_{i}(t+1)$ is found by

$$
\begin{array}{r}
a_{i}(t+1)=\arg \min _{x \in[-1,1]} g\left(a_{1}(t+1), \ldots, a_{i-1}(t+1), x,\right. \\
\left.a_{i+1}(t), \ldots, a_{N}(t)\right)
\end{array}
$$

while in the Jacobi iteration $a_{i}(t+1)$ is found by

$$
\begin{array}{r}
a_{i}(t+1)=\arg \min _{x \in[-1,1]} g\left(a_{1}(t), \ldots, a_{i-1}(t), x,\right. \\
\left.a_{i+1}(t), \ldots, a_{N}(t)\right)
\end{array}
$$

respectively, where $t$ is the stage (iteration) index. Both algorithms optimize one variable at a time to get to the optimum point of (6); however, (7) uses the current stage estimates of some of the users while (8) allows a parallel implementation. Through a straightforward derivation, it can be shown that the above iterations yield the following two-step algorithms. For each user $i$, the first step for the Gauss-Seidel iteration is

$$
\begin{aligned}
\hat{x}(t+1)=\frac{1}{\sqrt{q_{i}}}\left(y_{i}-\sum_{j=1}^{i-1} \sqrt{q_{j}}\right. & \Gamma_{j i} a_{j}(t+1) \\
& \left.\quad-\sum_{j=i+1}^{N} \sqrt{q_{j}} \Gamma_{j i} a_{j}(t)\right)
\end{aligned}
$$

and the first step for the Jacobi iteration is

$$
\hat{x}(t+1)=\frac{1}{\sqrt{q_{i}}}\left(y_{i}-\sum_{j=1, j \neq i}^{N} \sqrt{q_{j}} \Gamma_{j i} a_{j}(t)\right) .
$$

The second step for both algorithms is

$$
a_{i}(t+1)= \begin{cases}-1, & \hat{x}(t+1)<-1 \\ \hat{x}(t+1), & -1 \leq \hat{x}(t+1) \leq 1 \\ 1, & \hat{x}(t+1)>1 .\end{cases}
$$

At each stage, to get the estimate of each user's bit, both receivers use soft estimates of the bits to reconstruct the interference and subtract this estimate from the user's matched filter output, scale the result by the amplitude of the user, and project

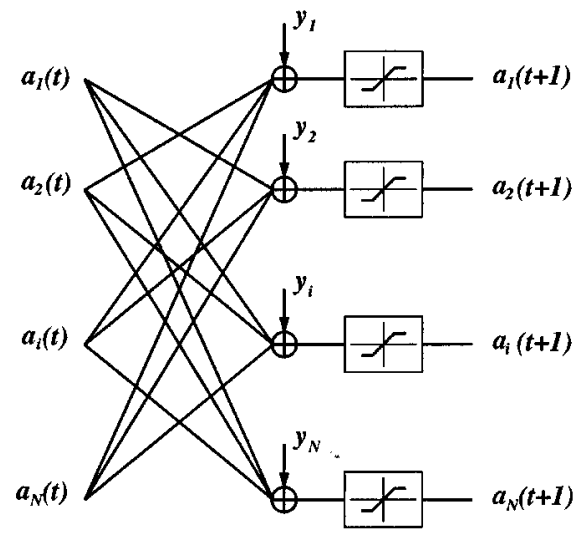

Fig. 3. Parallel soft interference canceler; see (10) and (11).

onto $[-1,1]$. The difference between the two is that while the Gauss-Seidel algorithm uses the available current stage estimates of the users, i.e., feedback from a group of users whose bit estimates are already computed, the Jacobi algorithm uses only bit estimates from the previous stage. In multiuser detection terms, the nonlinear Gauss-Seidel algorithm corresponds to the successive soft interference canceler (Fig. 2), whereas the nonlinear Jacobi algorithm corresponds to the parallel soft interference canceler (Fig. 3).

In the following, we establish the convergence of these two algorithms. For that, we use results from [14] and omit the proofs here. First, we state the following theorem for the convergence of the nonlinear Gauss-Seidel algorithm.

Theorem 1 [14, Proposition 3.9 of Sect. 3.3]: Suppose that $g: \mathcal{R}^{N} \rightarrow \mathcal{R}$ is continuously differentiable and convex on the set $X$ where $X$ is a Cartesian product of sets $X_{i}$ and each $X_{i}$ is a closed convex subset of $\mathcal{R}^{n_{i}}$. Furthermore, suppose that, for each $i, g$ is a strictly convex function of $x_{i}$, when the values of the other components of $x$ are held constant. Let $x(t)$ be the sequence generated by the nonlinear Gauss-Seidel algorithm, assumed to be well defined. Then every limit point of $\boldsymbol{x}(t) \mathrm{min}-$ imizes $g$ over $X$.

In our case, $g(\boldsymbol{a})=\boldsymbol{a}^{\top} \boldsymbol{R} \boldsymbol{a}-2 \boldsymbol{a}^{\top} \boldsymbol{\Lambda} \boldsymbol{y}$, and for a positive definite $\boldsymbol{\Gamma}$, the function $g(\boldsymbol{a})$ is convex in $\boldsymbol{a}$ and is strictly convex in each variable $a_{i}$ when the values of the other components of $a$ are held constant. The convex set $X=[-1,1]^{N}$ is in Cartesian product form. The convexity of $g(\boldsymbol{a})$ ensures the uniqueness of the convergence point which is the global minimum. The following corollary states the convergence condition for the successive soft interference canceler. 
Corollary 1: For a CDMA system with linearly independent signature sequences, the nonlinear Gauss-Seidel algorithm (the successive soft interference canceler) always converges to the minimizer of (6).

Theorem 1 shows that the nature of the problem at hand gives us the convergence of the Gauss-Seidel algorithm without any further restrictions. Unfortunately, this is not the case for the Jacobi algorithm. Convergence of the parallel soft interference canceler can be guaranteed under certain contraction assumptions as indicated by the following theorem. We first state the theorem and then derive the conditions for it to hold.

Theorem 2 [14, Proposition 3.10, Sect. 3.3]: Let $g: \mathcal{R}^{N} \rightarrow$ $\mathcal{R}$ be continuously differentiable, let $\gamma$ be a positive scalar, and suppose that the mapping $T: X \rightarrow \mathcal{R}^{N}$, defined by $T(x)=$ $\boldsymbol{x}-\gamma \nabla g(\boldsymbol{x})$, is a contraction with respect to the block maximum norm $\|\boldsymbol{x}\|_{\infty}^{w}=\max _{i}\left\|x_{i}\right\|_{i} / w_{i}$, where each $\|\cdot\|_{i}$ is the Euclidean norm on $\mathcal{R}^{n_{i}}$ and each $w_{i}$ is a positive scalar. Then, there exists a unique vector $\boldsymbol{x}^{*}$ which minimizes $g$ over $X$. Furthermore, the sequence $\boldsymbol{x}(t)$ generated by either the Gauss-Seidel or the Jacobi algorithms converges to $\boldsymbol{x}^{*}$ geometrically.

For our problem, $g(\boldsymbol{x})=\boldsymbol{x}^{\top} \boldsymbol{R} \boldsymbol{x}-2 \boldsymbol{x}^{\top} \boldsymbol{\Lambda} \boldsymbol{y}$ and $T(\boldsymbol{x})$ is given by

$$
T(\boldsymbol{x})=\boldsymbol{x}-\gamma(2 \boldsymbol{R} \boldsymbol{x}-2 \boldsymbol{\Lambda} \boldsymbol{y})=(\boldsymbol{I}-2 \gamma \boldsymbol{R}) \boldsymbol{x}+2 \gamma \boldsymbol{\Lambda} \boldsymbol{y}
$$

In order to guarantee the convergence of the Jacobi algorithm (parallel soft interference canceler), we need $T(\boldsymbol{x})$ to be a contraction mapping with respect to a block maximum norm. For any matrix $A$, the block maximum norm $\|A\|_{\infty}^{w}$ is defined [14] as $\|\boldsymbol{A}\|_{\infty}^{w}=\max _{i}\left(1 / w_{i}\right) \sum_{j}\left|A_{i j}\right| w_{j}$. A mapping $T(\boldsymbol{x})$ is called a contraction mapping with respect to a block maximum norm $\|\cdot\|_{\infty}^{w}$, if and only if (iff) $T(\boldsymbol{x})$ satisfies

$$
\left\|T(\boldsymbol{x})-T\left(\boldsymbol{x}^{\prime}\right)\right\|_{\infty}^{w}<\alpha\left\|\boldsymbol{x}-\boldsymbol{x}^{\prime}\right\|_{\infty}^{w}
$$

for any $\boldsymbol{x}$ and $\boldsymbol{x}^{\prime}$, for some $\alpha \in[0,1)$. For the mapping in (12), this condition is equivalent to

$$
\|\boldsymbol{I}-2 \gamma \boldsymbol{R}\|_{\infty}^{w}<1
$$

If (14) holds, then the nonlinear Jacobi algorithm converges by Theorem 2. However, for a given CDMA system, it may be difficult to verify whether (14) holds. Our aim is to derive an easier condition to check for the convergence of the nonlinear Jacobi algorithm. It is desirable for this condition to be independent of the received powers of the users $\gamma$ and a particular block maximum norm $\|\cdot\|_{\infty}^{w}$. The following Lemma states the condition for the convergence of the nonlinear Jacobi algorithm (parallel soft interference canceler) in terms of only the cross-correlation matrix.

Lemma 1: For a CDMA system with linearly independent signature sequences, the nonlinear Jacobi algorithm (parallel soft interference canceler) converges to the minimizer of (6) if

$$
\rho(\overline{\boldsymbol{\Gamma}})<1
$$

where $\overline{\boldsymbol{\Gamma}}$ is constructed from $\boldsymbol{\Gamma}$ as $\bar{\Gamma}_{i j}=\left|\Gamma_{i j}\right|$ if $i \neq j$, and $\bar{\Gamma}_{i i}=0$, and $\rho(\cdot)$ denotes the maximum eigenvalue of a matrix.
Proof-Lemma 1: Using the definition of the block maximum norm, it can be shown that, for a sufficiently small $\gamma,(14)$ is equivalent to

$$
\max _{i}\left\{\left(1-2 \gamma R_{i i}\right)+2 \gamma \sum_{j \neq i}\left|R_{i j}\right| \frac{w_{j}}{w_{i}}\right\}<1
$$

which is satisfied iff

$$
-R_{i i}+\sum_{j \neq i}\left|R_{i j}\right| \frac{w_{j}}{w_{i}}<0 \text { for all } i .
$$

Substituting $R_{i j}=\sqrt{q_{i}} \sqrt{q_{j}} \Gamma_{i j}$ into (17) and noting $\Gamma_{i i}=1$, we arrive at

$$
\sum_{j \neq i}\left|\Gamma_{i j}\right| \frac{\sqrt{q_{j}}}{\sqrt{q_{i}}} \frac{w_{j}}{w_{i}}<1 \text { for all } i .
$$

Let $\bar{w}_{j}=w_{j} \sqrt{q_{j}}$. Using the definition of $\overline{\boldsymbol{\Gamma}},(18)$ is equivalent to

$$
\|\overline{\boldsymbol{\Gamma}}\|_{\infty}^{\bar{w}}<1
$$

Finally, [14, Sect. 2.6, Corollary 6.2] states that (19) is satisfied iff $\rho(\overline{\boldsymbol{\Gamma}})<1$, completing the proof.

To summarize, for a given cross-correlation matrix, one can check if (15) holds by constructing $\overline{\boldsymbol{\Gamma}}$ and finding its maximum eigenvalue. If it does, then by Theorem 2, the parallel soft interference canceler is guaranteed to converge to the minimizer of (6).

In the proof of Lemma 1, we arrived at (15) from its equivalent condition given in (19). An interesting observation is that, choosing $\bar{w}_{i}=1$ for all $i,(19)$ reduces to a diagonal dominance condition, $\sum_{j \neq i}\left|\Gamma_{i j}\right|<\Gamma_{i i}$, for all $i$. This is clearly much easier to verify, however it is a more lenient condition for convergence since a particular set of $\bar{w}$ values are used. It is possible for the parallel soft interference canceler to converge for a system with its cross-correlation matrix satisfying (15) even if is not diagonally dominant (see Section VI).

It is also interesting to note that both the diagonal dominance condition mentioned above and the maximum eigenvalue condition in (15) are satisfied for a system where users' signatures are shifted versions of a basic $m$-sequence. In this case, $\Gamma_{i j}=-1 / G, i \neq j$, and $\rho(\overline{\boldsymbol{\Gamma}})=(N-1) / G$, and $\rho(\overline{\boldsymbol{\Gamma}})<1$ as long as $N \leq G$ which by definition is the case. Diagonal dominance condition is also equivalent to $(N-1) / G<1$ for $m$-sequences. Thus, if these sequences are used, both Jacobi and Gauss-Seidel algorithms, i.e., parallel and successive soft interference cancelers, converge to the minimizer of (6).

In general, it takes more than one iteration for either algorithm to converge and thus the resulting receivers are multi-stage receivers. Multi-stage receivers are familiar in multiuser detection. In [5], hard decision bit estimates are used to reconstruct and subtract the interference for each user. The receiver is implemented in a parallel fashion as in (10) and is not convergent. In [16], the authors proposed a class of receivers based on the SAGE algorithm, one of which is the successive multistage receiver (9) and argues that the SAGE-based hard decision multistage receiver is convergent even when its parallel counterpart is not. The soft decision versions of these multistage receivers, 
i.e., (9) and (10), are proposed in [16] and [17]. They are termed as receivers with linear clippers. By representing these receivers in the form of iterative nonlinear programming algorithms, we have shown that both of these soft decision receivers, i.e., the parallel and the successive soft multi-stage interference cancelers, if they converge, converge to the same point which is the minimizer of (6). We have observed that the Gauss-Seidel algorithm has a more lenient requirement in order to converge, i.e., linear independence of the signature sequences is sufficient, while the condition that guarantees the Jacobi algorithm to converge is more strict and is given in (15). Typically, Gauss-Seidel type iterations have faster convergence since they use the newest estimates. On the other hand, Jacobi type iterations can be executed in a completely parallel fashion since they do not require feedback from the current stage estimate of any user. Note that, if Theorem 2 is valid, any combination of the two algorithms also converges to the minimum of (6), i.e., some users can use the successive soft multi-stage receivers and others can use the parallel soft multi-stage receivers.

It is worthwhile to note that one can implement the decorrelator given by (5) iteratively. Gauss-Seidel and Jacobi algorithms that converge to (5) can be found to be the algorithms derived in this section without the second stage $[-1,1]$ clippers. It is also possible to derive Gauss-Seidel and Jacobi iterations that converge to the MMSE detector [4] which estimates the bits by taking the sign of

$$
\overline{\boldsymbol{a}}=\boldsymbol{\Lambda}^{-1}\left(\boldsymbol{\Gamma}+\sigma^{2} \boldsymbol{\Lambda}^{-2}\right)^{-1} \boldsymbol{y} .
$$

It can be shown that the resulting algorithms differ from (9) and (10) only in the scaling factor. Specifically, we have

$$
\begin{array}{r}
\hat{x}(t+1)=\frac{\sqrt{q_{i}}}{q_{i}+\sigma^{2}}\left(y_{i}-\sum_{j=1}^{i-1} \sqrt{q_{j}} \Gamma_{j i} a_{j}(t+1)\right. \\
\left.-\sum_{j=i+1}^{N} \sqrt{q_{j}} \Gamma_{j i} a_{j}(t)\right) \\
\hat{x}(t+1)=\frac{\sqrt{q_{i}}}{q_{i}+\sigma^{2}}\left(y_{i}-\sum_{j=1, j \neq i}^{N} \sqrt{q_{j}} \Gamma_{j i} a_{j}(t)\right)
\end{array}
$$

for the Gauss-Seidel and the Jacobi iterations, respectively. Further, these iterations can be followed by the $[-1,1]$ clippers. The resulting receivers converge to the minimizer of the following quadratic program which is similar to (6) where the matrix $\boldsymbol{R}$ is replaced by $\boldsymbol{R}+\sigma^{2} \boldsymbol{I}$

$$
\min _{\boldsymbol{a} \in[-1,1]^{N}} \boldsymbol{a}^{\top}\left(\boldsymbol{R}+\sigma^{2} \boldsymbol{I}\right) \boldsymbol{a}-2 \boldsymbol{a}^{\top} \boldsymbol{\Lambda} \boldsymbol{y} .
$$

Note that this minimization problem when defined on the corners of the unit hypercube, i.e., $\boldsymbol{a} \in\{-1,1\}^{N}$, is exactly the same as the original OMUD problem in (3), since on the corners of the unit hypercube the additional term $\sigma^{2} \boldsymbol{a}^{\top} \boldsymbol{a}$ in the cost function is independent of the choice of $\boldsymbol{a}$. An equivalent of the convergence condition (15) can be easily derived for the MMSE type receivers with clippers using the same steps we followed in this section. The algorithms without the clippers, i.e., the algorithms that converge to the decorrelating or the MMSE detectors, correspond to linear Gauss-Seidel and Jacobi iterations. For these iterations, Theorem 2 still gives the sufficient condition for convergence. However, less stringent conditions which are necessary and sufficient are available for this case. For the decorrelator, for example, using [18, Theorem 3.3 of Sect. 4.3], one can show that the necessary and sufficient condition for convergence of the Jacobi algorithm without the clippers is

$$
\rho(\tilde{\boldsymbol{\Gamma}})<1
$$

where $\tilde{\boldsymbol{\Gamma}}$ is the matrix consisting of the off-diagonal elements of $\boldsymbol{\Gamma}$, i.e., $\tilde{\Gamma}_{i j}=\Gamma_{i j}$, if $i \neq j$, and $\tilde{\Gamma}_{i i}=0$. For the MMSE detector, the necessary and sufficient condition is found by replacing $\boldsymbol{\Gamma}$ with $\boldsymbol{\Gamma}+\sigma^{2} \boldsymbol{\Lambda}^{-2}$. To see why (24) is less stringent than (15), first observe that $\overline{\boldsymbol{\Gamma}}=|\tilde{\boldsymbol{\Gamma}}|$, and by definition of the $\|\cdot\|_{\infty}^{w}$ norm of a matrix, $\|\tilde{\boldsymbol{\Gamma}}\|_{\infty}^{w}=\|\overline{\boldsymbol{\Gamma}}\|_{\infty}^{w}$. We can then use [14, Proposition $6.2(\mathrm{e})$ of Sect. 2.6] which states that $\rho(\mathbf{A}) \leq\|\mathbf{A}\|_{\infty}^{w}$ for any matrix $\mathbf{A}$. Thus, the following relationship holds:

$$
\rho(\tilde{\boldsymbol{\Gamma}}) \leq\|\overline{\boldsymbol{\Gamma}}\|_{\infty}^{w} .
$$

This means that if (19) [or equivalently (15)] holds then (24) is guaranteed to hold, which makes it less stringent.

Finally, we should emphasize that the implementations discussed here are not the unique way of solving for the minimizer of (6). There are other nonlinear programming methods that yield iterative algorithms whose BER performance matches that of the soft interference cancelers.

\section{GENERALIZEd MMSE DETECTOR}

The constraint on each $a_{i} \in\{-1,1\}$ is equivalent to $a_{i}^{2}=1$ which implies $\boldsymbol{a}^{\top} \boldsymbol{a}=N$ at any feasible point for OMUD. Relaxing this set to $\boldsymbol{a}^{\top} \boldsymbol{a} \leq N$, i.e., to the smallest sphere that contains the corners of the unit hypercube, results in the following optimization problem:

$$
\min _{\mathbf{a}^{\top} \boldsymbol{a} \leq N} \boldsymbol{a}^{\top} \boldsymbol{R} \boldsymbol{a}-2 \boldsymbol{a}^{\top} \boldsymbol{\Lambda} \boldsymbol{y}
$$

Since (26) minimizes a convex function over a convex set, it has a unique minimum and iterative algorithms such as gradient descent can be employed to find this minimum [19]. Further, the convex duality theorem [19, Theorem 14.6] ensures that no duality gap exists and one can solve for the dual problem instead. Since (26) has a single constraint, there is only one dual variable. Thus, a simpler iterative algorithm can be found by solving the dual problem as outlined below.

The Lagrangian dual function can be expressed as

$$
\mathcal{L}(\boldsymbol{a}, \lambda)=\boldsymbol{a}^{\top} \boldsymbol{R} \boldsymbol{a}-2 \boldsymbol{a}^{\top} \boldsymbol{\Lambda} \boldsymbol{y}+\lambda\left(\boldsymbol{a}^{\top} \boldsymbol{a}-N\right)
$$

which is to be minimized over $\boldsymbol{a}$ and maximized over $\lambda \geq 0$. Solving for $\boldsymbol{a}$ in terms of $\lambda$ and substituting back, we obtain

$$
\max _{\lambda \geq 0}-\boldsymbol{y}^{\top} \boldsymbol{\Lambda}(\boldsymbol{R}+\lambda \boldsymbol{I})^{-1} \boldsymbol{\Lambda} \boldsymbol{y}-\lambda N
$$

which is a one-dimensional optimization problem. The optimization problem (28) entails the optimization of a rational function of the variable $\lambda$ and can be solved with a variety 
of iterative algorithms [19]. A simple unconstrained gradient descent algorithm is guaranteed to converge for a sufficiently small step size $\mu$ which can then be projected onto the positive axis. The algorithm is

$$
\bar{\lambda}(t+1)=\bar{\lambda}(t)+\mu\left(\boldsymbol{y}^{\top} \boldsymbol{\Lambda}(\boldsymbol{R}+\bar{\lambda}(t) \boldsymbol{I})^{-2} \boldsymbol{\Lambda} \boldsymbol{y}-N\right)
$$

which converges to $\bar{\lambda}$ for a reasonable choice of $\mu$. The maximizer of (28) is given by

$$
\lambda^{*}=\max (0, \bar{\lambda}) .
$$

Then, the unique minimizer of (26) can be found to be

$$
\boldsymbol{a}^{*}=\left(\boldsymbol{R}+\lambda^{*} \boldsymbol{I}\right)^{-1} \boldsymbol{\Lambda} \boldsymbol{y}=\boldsymbol{\Lambda}^{-1}\left(\boldsymbol{\Gamma}+\lambda^{*} \boldsymbol{\Lambda}^{-2}\right)^{-1} \boldsymbol{y} .
$$

The form of this solution whose sign is the estimate of the bit vector is also familiar because of its similarity to the MMSE detector [4]. Thus, we term the relaxation (26) the generalized MMSE (GMMSE) solution. When $\lambda^{*}=\sigma^{2}$, (31) reduces to the MMSE detector. Note that, since $\lambda^{*}$ is a function of $\boldsymbol{y}$, the GMMSE solution results in a nonlinear multiuser detector in contrast to the MMSE detector. On the other hand, the knowledge of the noise power value $\left(\sigma^{2}\right)$ is not necessary for the GMMSE detector whereas the MMSE detector requires this knowledge if training or blind adaptation is not desired [4], [20].

The GMMSE detector is also an iterative detector since $\lambda^{*}$ has to be found iteratively. However, since the iterations are in one dimension, they are expected to converge more quickly compared to multidimensional algorithms.

\section{RESULTS}

Since the probability of bit error expressions are not analytically tractable for an arbitrary number of users and iterations, we have simulated the bit error performance of the detectors investigated in this work. All iterative detectors (multistage soft cancelers and the GMMSE) are evaluated at their convergence points. The simulation scenarios are representative examples of highly loaded systems where implementation of multiuser detectors is well justified for improved system performance. The system size is kept modest for the sake of computational feasibility of the simulations.

The first system simulated is an $N=4$ user synchronous system with processing gain $G=7$ that uses Gold sequences with the cross-correlation matrix

$$
\boldsymbol{\Gamma}=\frac{1}{7}\left[\begin{array}{rrrr}
7 & -1 & 3 & 3 \\
-1 & 7 & 3 & -1 \\
3 & 3 & 7 & -1 \\
3 & -1 & -1 & 7
\end{array}\right]
$$

Note that, for this cross-correlation matrix, the diagonal dominance condition is not satisfied. However, the parallel soft interference canceler is still guaranteed to converge because $\rho(\overline{\mathbf{\Gamma}})=$ $0.8747<1$. Fig. 4 shows the probability of bit error for the third user when that user has a 6-dB SNR and all the interferers have a common SNR (in $\mathrm{dB}$ ) that is varied. We observe that the soft interference cancelers [(9)-(11)] have almost invariable performance versus interference strength. We note that the per-

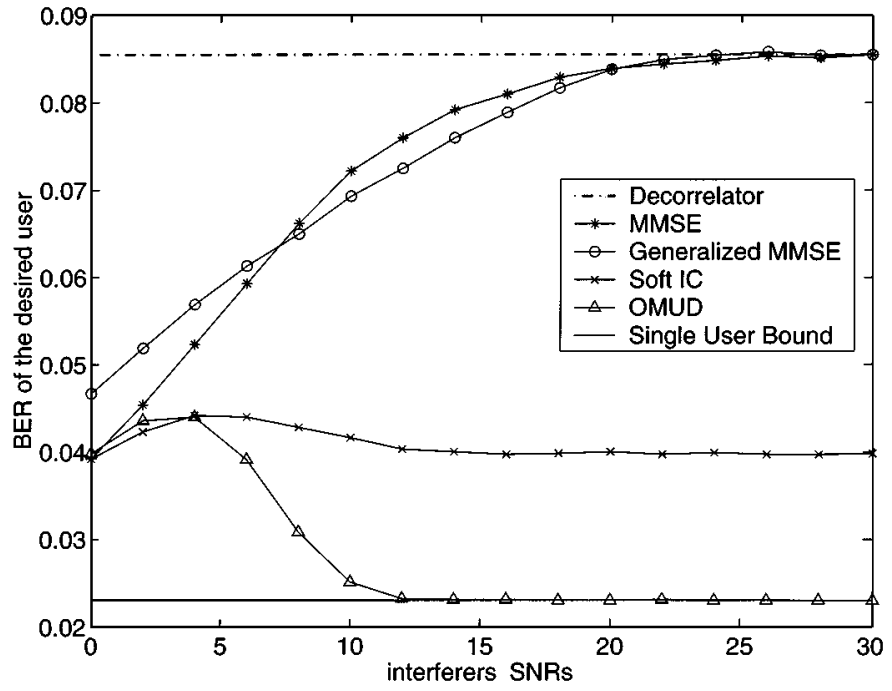

Fig. 4. Comparison of error probabilities of near-optimum multiuser detectors, near-far scenario, desired user at a 6-dB SNR. $G=7, N=4$, Gold sequences.

formance of the GMMSE detector is similar to that of the linear MMSE detector. In particular, we observe that the GMMSE detector has the same trend of approaching the decorrelator performance as the MMSE detector when the interference dominates the noise. Although the performance of the GMMSE detector is not amenable to analysis due to its nonlinear nature, its asymptotic behavior in the severe near-far regime is identical to that of the MMSE detector due to the structural similarity of the two detectors. Both the noise level $\sigma^{2}$ in the MMSE detector expression and the optimum Lagrange multiplier $\lambda^{*}$ in the GMMSE detector expression become insignificant as the interference level goes to infinity. This causes both detectors' performance to approach the decorrelator performance in this asymptotic regime.

Next, we simulated the bit error performance of a user in an $N=7$ user synchronous system with processing gain $G=$ 7 that uses $m$-sequences, i.e., $\Gamma_{i j}=-1 / 7 j \neq i$, and have observed similar trends (Fig. 5). As mentioned in Section IV, $m$-sequences satisfy the diagonal dominance condition and thus the parallel interference canceler is guaranteed to converge.

Our last simulation set up is of an $N=3$ user system with processing gain $G=7$ that uses the set of Gold sequences which constitute the rows of the following matrix $S$ :

$$
\boldsymbol{S}=\left[\begin{array}{rrrrrrr}
1 & -1 & -1 & -1 & -1 & 1 & -1 \\
-1 & 1 & 1 & -1 & -1 & 1 & 1 \\
1 & -1 & -1 & 1 & 1 & 1 & -1
\end{array}\right]
$$

First, we simulated the synchronous system performance where the cross-correlation matrix is

$$
\boldsymbol{\Gamma}=\frac{1}{7}\left[\begin{array}{rrr}
7 & -1 & 3 \\
-1 & 7 & -5 \\
3 & -5 & 7
\end{array}\right]
$$

Once again, the diagonal dominance condition is not satisfied, however, $\rho(\overline{\mathbf{\Gamma}})=0.9008<1$ and the parallel soft interference canceler is guaranteed to be convergent. The resulting BER of the first user is given in Fig. 6 . 


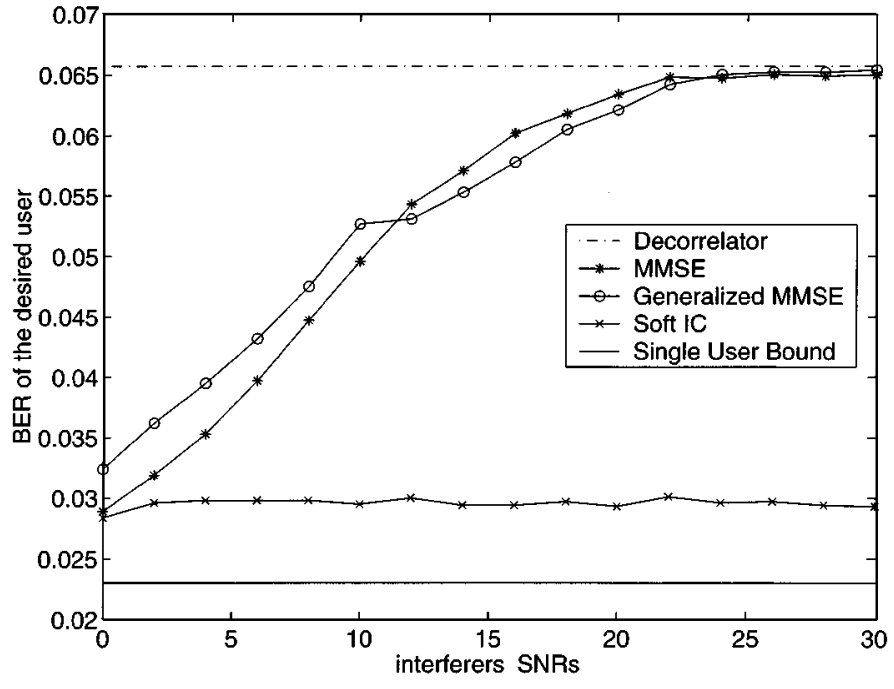

Fig. 5. Comparison of error probabilities of near-optimum multiuser detectors, near-far scenario, desired user at a 6-dB SNR. $G=7, N=7, M$-sequences.

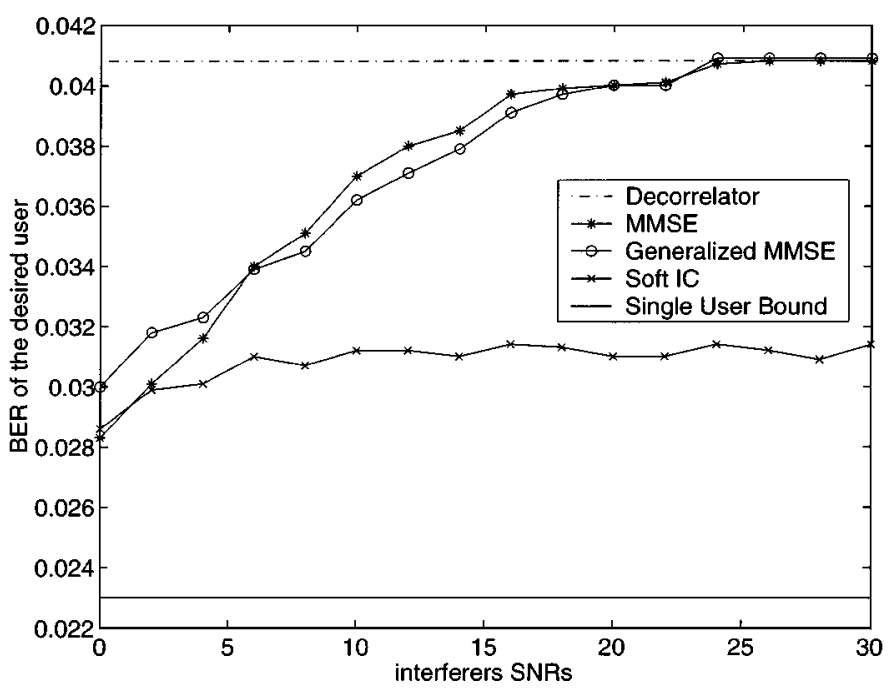

Fig. 6. Comparison of error probabilities of near-optimum multiuser detectors, near-far scenario, desired user at a 6-dB SNR. $G=7, N=3$, Gold sequences.

For this synchronous system, we also investigated the performance of the nonadaptive MMSE detector and the GMMSE detector when the Gaussian noise variance is estimated. A likely scenario for this situation is a multicell system where the intra-cell multiuser detection is performed and the out-of-cell interference can be accurately described by additive Gaussian noise. As mentioned in Section V, the GMMSE detector does not require the value of the noise variance. The MMSE detector on the other hand requires this value, see (20), and thus is potentially vulnerable to imperfect estimates. Fig. 7 shows the bit error rate of the desired user versus the estimation error of the noise variance in $\mathrm{dB}$, when the desired user has $6 \mathrm{~dB}$ and the interferers have $10 \mathrm{~dB}$ SNR values. Note that, the MMSE performance with $0 \mathrm{~dB}$ estimation error corresponds to the performance of the MMSE detector with the perfect noise variance estimate. As expected, the nonadaptive MMSE detector is sensitive to noise variance estimation errors. In particular, when the noise variance is severely underestimated,

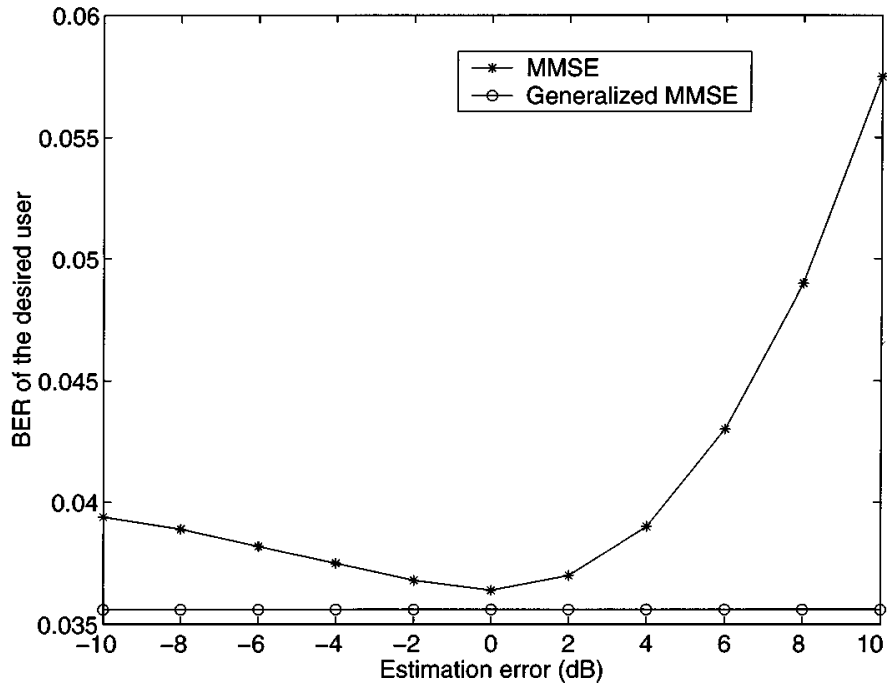

Fig. 7. Comparison of error probabilities of GMMSE and MMSE detectors under imperfect estimates of aggregate Gaussian noise variance; plotted is the desired user bit error rate versus the estimation error in $\mathrm{dB}$. Desired user at $6 \mathrm{~dB}$, interferers at $10 \mathrm{~dB}$ SNR. Same system as in Fig. 6.

the performance of the MMSE detector approaches that of the decorrelator which is 0.0408 ; when the noise variance is overestimated the performance gradually gets worse and closer to that of a single user receiver (matched filter) which for this system is 0.1412 . These two observations are easily justified by inspecting the structure of the MMSE detector in (20). When a small noise variance is used, the detector resembles a decorrelator, whereas when a very large noise variance is used, the second term in the inverse transformation becomes dominant, i.e., the detector is similar to a matched filter. Since the GMMSE performance is unaffected by these estimation errors, it is of value to be used under such a scenario.

Lastly, we considered the performance of the same system where users transmit $M$-bit packets in an asynchronous fashion. The relative delays of the users are assumed to be less than the bit duration. Note that in this case the receiver should decode all bits of all the users jointly by observing the received signal for the entire packet transmission duration of all the users. The sufficient statistics to decode the bits are obtained by passing the received signal through the matched filter of each user in each of its bit transmission intervals. This way, in effect, each user becomes equivalent to $M$ effective independent users for the OMUD problem. To explain this point further, consider that the transmission delays of the users are sorted in increasing order, the $i$ th user has a transmission delay of $d_{i}$ chips and $d_{1}=0$. The observation interval then becomes $M G+d_{N}$ chips, or $(M G+$ $\left.d_{N}\right) T_{c}$ seconds where $T_{c}$ is the chip duration; that is, an interval large enough to capture the entire packet of the latest arriving user. Bit $k$ of user $i$ is received in the interval $[((k-1) G+$ $\left.\left.d_{i}\right) T_{c},\left(k G+d_{i}\right) T_{c}\right]$. The corresponding matched filter for this bit is the signature of the $i$ th user in this interval padded with zeros from left and right for the rest of the observation interval. The resulting cross-correlation matrix, $\boldsymbol{\Gamma}$ is $N M \times N M$ and the OMUD is formally stated as

$$
\boldsymbol{a}^{*}=\arg \min _{\boldsymbol{a} \in\{-1,1\}^{N M}} \boldsymbol{a}^{\top} \boldsymbol{R} \boldsymbol{a}-2 \boldsymbol{a}^{\top} \boldsymbol{\Lambda} \boldsymbol{y}
$$




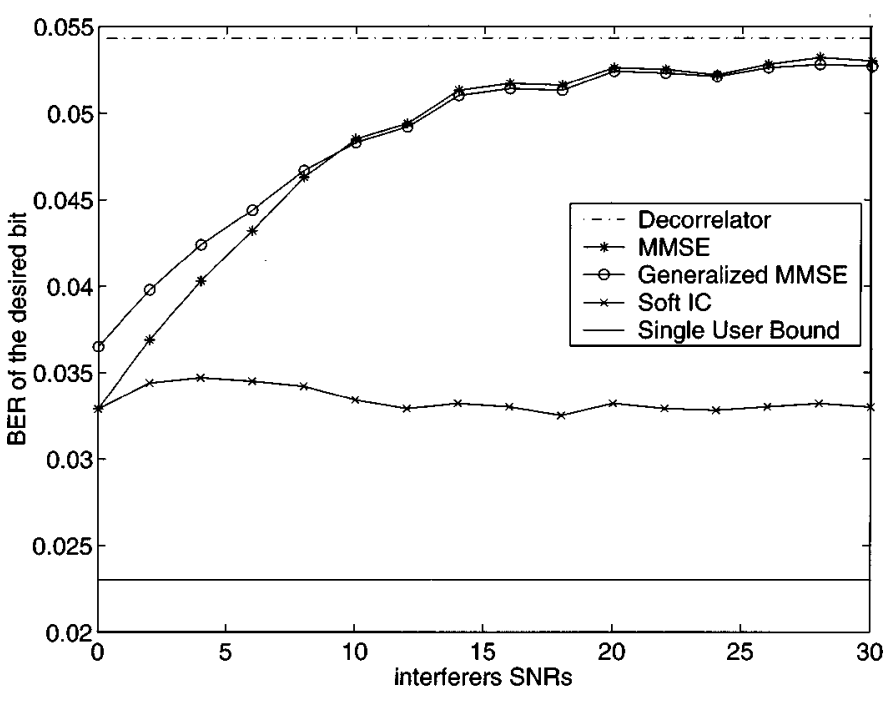

Fig. 8. Comparison of error probabilities of near-optimum multiuser detectors for an asynchronous system with $M$-bit packet transmission, near-far scenario, desired user at $6 \mathrm{~dB}$ SNR. $G=7, N=4, M=3$, same set of Gold sequences as in Fig. 6. The error probability of the desired user's middle bit is plotted.

where $\boldsymbol{R}$ again is given by $\boldsymbol{R}=\boldsymbol{\Lambda} \boldsymbol{\Gamma} \boldsymbol{\Lambda}$ and $\sqrt{q_{i}}$ appears as $\Lambda_{k k}$ for $k=(i-1) M+1, \ldots, i M$. The $k$ th bit of user $i$ is denoted by $a_{(i-1) M+k}$. Fig. 8 shows the error rate for the middle bit of the first user when $M=3$ bits. In this particular example, the delays of the users are $0,2,5$ chips respectively and the resulting $9 \times 9$ cross correlation matrix $\boldsymbol{\Gamma}$ satisfies the diagonal dominance condition. Figs. 6 and 8 confirm our observations from the previous experiments regarding the close performance of MMSE and GMMSE and the near-far resistant performance of the soft interference cancelers.

\section{CONCLUSION}

In this paper, we have shown that many popular suboptimum detectors are devices that attempt to approximate the solution of the joint minimum BER detector (OMUD). Although it is analytically hard to characterize exactly how closely they approximate the OMUD cost function, we have observed that they achieve near-optimum cost values. Consequently, the near-optimum BER performances of these detectors are not surprising. The nonlinear programming approach helped us to identify the convergence conditions of multistage soft interference cancelers. We have also proposed and devised a simple iterative nonlinear detector with similar performance to the MMSE detector. The generalized MMSE detector, in contrast to the nonadaptive version of the MMSE detector, does not require the knowledge of the ambient noise power level. Thus, it can be used in scenarios where adaptive or blind adaptive detection is not suitable, for instance when the channel is changing rapidly, and the ambient noise power is unknown. A likely scenario is a multicell setting where only in-cell system information is available, and thus in-cell multiuser detection is feasible. In this case, the out-of-cell interference manifests itself by amplifying the Gaussian noise and the GMMSE detector results in MMSE-like performance without the need to estimate this level.

Finally, we note that the nonlinear programming approach can be used to derive other types of detectors under different channel fading conditions as well. For instance, it is easy to show that the hypercube type relaxation is the optimum detector when the uplink gains of the users are assumed completely random in $[0,1]$ and unknown [21]. For real channels, the uplink gains are likely to have much smaller values than 1 , and have different distributions depending on the channel fading conditions. Using this fact, detectors for channels with uncertainties can be designed with smaller box boundaries. Thus, this approach can be useful in designing joint bit detectors and channel estimators under different conditions.

\section{REFERENCES}

[1] S. Verdú, Multiuser Detection. Cambridge, U.K.: Cambridge Univ. Press, 1998.

[2] — - "Computational complexity of multiuser detection," Algorithmica, vol. 4, no. 4, pp. 303-312, 1989.

[3] R. Lupas and S. Verdú, "Linear multiuser detectors for synchronous code-division multiple-access channels," IEEE Trans. Inform. Theory, vol. 35, pp. 123-136, Jan. 1989.

[4] U. Madhow and M. L. Honig, "MMSE interference suppression for direct-sequence spread-spectrum CDMA," IEEE Trans. Commun., vol. 42, pp. 3178-3188, Dec. 1994.

[5] M. K. Varanasi and B. Aazhang, "Multistage detection in asynchronous code-division multiple-access communications," IEEE Trans. Commun., vol. 38, pp. 509-519, Apr. 1990.

[6] A. Duel-Hallen, "Decorrelating decision-feedback multiuser detector for synchronous code-division multiple-access channels," IEEE Trans. Commun., vol. 41, pp. 285-290, Feb. 1993.

[7] M. K. Varanasi and B. Aazhang, "Near-optimum detection in synchronous code-division multiple-access systems," IEEE Trans. Commun., vol. 39, pp. 725-736, May 1991.

[8] P. Patel and J. Holtzman, "Analysis of a simple successive interference cancellation scheme in a DS/CDMA system," IEEE J. Select. Areas Commun., vol. 12, pp. 796-807, June 1994.

[9] S. Verdú, "Minimum probability of error for asynchronous Gaussian multiple-access channels," IEEE Trans. Inform. Theory, vol. IT-32, pp. 85-96, Jan. 1986.

[10] S. Ulukus and R. D. Yates, "Optimum multiuser detection is tractable for synchronous CDMA systems using $M$-sequences," IEEE Commun. Lett., vol. 2, pp. 89-91, Apr. 1998.

[11] C. Sankaran and A. Ephremides, "Solving a class of optimum multiuser detection problems with polynomial complexity," IEEE Trans. Inform. Theory, vol. 44, pp. 1958-1961, Sept. 1998.

[12] U. Fawer and B. Aazhang, "A multiuser receiver for code division multiple access communications over multipath channels," IEEE Trans. Commun., vol. 43, pp. 1556-1565, Feb./Mar./Apr. 1995.

[13] X. Wang and H. V. Poor, "Space-time multiuser detection in multipath CDMA channels," IEEE Trans. Signal Processing, vol. 47, pp. 2356-2374, Sept. 1999.

[14] D. Bertsekas and J. Tsitsiklis, Parallel and Distributed Computation. Englewood Cliffs, NJ: Prentice-Hall, 1989.

[15] P. Hansen, "Methods of nonlinear 0-1 programming," in Annals of Discrete Mathematics 5: Discrete Optimization II, P. L. Hammer, E. L. Johnson, and B. H. Korte, Eds. Amsterdam, The Netherlands: North Holland, 1979.

[16] L. B. Nelson and H. V. Poor, "Iterative multiuser receivers for CDMA channels: An EM-based approach," IEEE Trans. Commun., vol. 44, pp. 1700-1710, Dec. 1996.

[17] X. Zhang and D. Brady, "Asymptotic multiuser efficiencies for decisiondirected multiuser detectors," IEEE Trans. Inform. Theory, vol. 44, pp. 502-515, Mar. 1998.

[18] D. M. Young, Iterative Solution of Large Linear Systems. New York: Academic, 1971.

[19] S. G. Nash and A. Sofer, Linear and Nonlinear Programming. New York: McGraw-Hill, 1996.

[20] M. Honig, U. Madhow, and S. Verdú, "Blind adaptive multiuser detection,” IEEE Trans. Inform. Theory, vol. 41, pp. 944-960, July 1995.

[21] A. Yener, R. D. Yates, and S. Ulukus, "A nonlinear programming approach to CDMA multiuser detection," in 33rd Asilomar Conf. on Signals, Systems and Computers, Oct. 1999. 


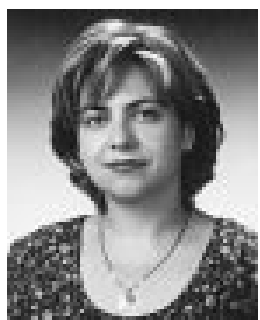

Aylin Yener (S'91-M'00) received the B.S. degrees in electrical and electronics engineering and in physics from Boğaziçi University, Istanbul, Turkey, in 1991 and the M.S. and Ph.D. degrees in electrical and computer engineering from Rutgers University, NJ, in 1994 and 2000, respectively.

During her Ph.D. studies, she was with Wireless Information Network Laboratory (WINLAB) in the Department of Electrical and Computer Engineering at Rutgers University, NJ. Between the fall of 2000 and the fall of 2001, she was with the Electrical Engineering and Computer Science Department at Lehigh University, Bethlehem, PA, where she was a P.C. Rossin Assistant Professor. Currently, she is with the Electrical Engineering Department at The Pennsylvania State University, University Park, as an Assistant Professor. Her research interests include performance enhancement of CDMA systems, wireless communication theory, and wireless networking in general.

Dr. Yener is an Associate Editor of the IEEE Transactions ON WIRELESS COMMUNICATIONS.

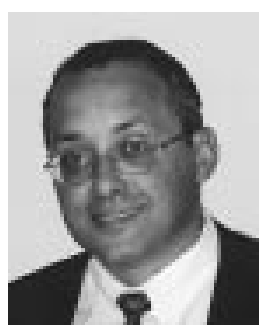

Roy D. Yates (M'91) received the B.S.E. degree from Princeton University, Princeton, NJ, in 1983 and the S.M. and Ph.D. degrees from the Massachusetts Institute of Technology, Cambridge, in 1986 and 1990, respectively, all in electrical engineering.

Since 1990, he has been with the Wireless Information Networks Laboratory (WINLAB) and the Electrical and Computer Engineering Department at Rutgers University, Piscataway, NJ. Presently, he serves as the Associate Director of WINLAB and Professor in the Electrical and Computer Engineering Department. He is a co-author (with David Goodman) of the text Probability and Stochastic Processes: A Friendly Introduction for Electrical and Computer Engineers (New York: Wiley). His research interests include power control, interference suppression, and media access protocols for wireless communications systems.

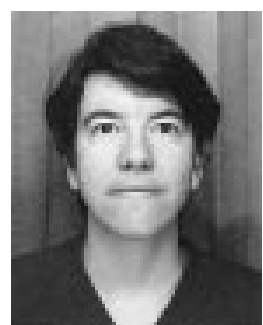

Sennur Ulukus (S'90-M'98) received the B.S. and M.S. degrees in electrical and electronics engineering from Bilkent University, Ankara, Turkey, in 1991 and 1993, respectively, and the Ph.D. degree in electrical and computer engineering from Rutgers University, Piscataway, NJ, in 1998.

During her Ph.D. studies, she was with the Wireless Information Network Laboratory (WINLAB), Rutgers University. From 1998 to 2001, she was a Senior Technical Staff Member at AT\&T Labs-Research in NJ. Since August 2001, she has been an Assistant Professor in the Department of Electrical and Computer Engineering at University of Maryland at College Park, where she also holds a joint appointment with the Institute for Systems Research. Her research interests are in wireless communication theory and networking, communication theory and information theory. 